\title{
Óbito Fetal em Microrregião de Minas Gerais: Causas e Fatores Associados
}

\author{
Stillbirth in a Microrregion of Minas Gerais State: \\ Causes and Associated Factors
}

Nestor Nurdan, Rosiane Mattar, Luiz Camano

\begin{abstract}
RESUM0
Objetivos: estudar as causas e fatores associados ao óbito fetal.

Métodos: estudo epidemiológico descritivo, que incluiu 190 casos de perdas fetais entre 11.825 gestantes que parturiram nos dois únicos hospitais (Casa de Saúde Divino Espirito Santo e Hospital Nossa Senhora Auxiliadora) de Caratinga, MG, no periodo de $1^{\circ}$ de janeiro de 1995 a 30 de abril de 2000. As variáveis investigadas foram: o número de gestações, a idade gestacional no momento da internação hospitalar, a época de ocorrência do decesso fetal em relação ao parto e a causa do óbito fetal. Não havendo grupo controle comparativo, utilizou-se estatística descritiva com tabelas, porcentagens e médias aritméticas.

Resultados: dentre as 189 gestantes com morte fetal, 77 eram primigestas e 76 tinham entre $2 e$ 5 gestações. A idade gestacional variou de 20 a 37 semanas. Em relação ao parto, a perda fetal ocorreu no periodo anteparto em 164 dos 190 casos. As causas mais freqüentes encontradas para a morte foram: DPP em 35, anomalia fetal em 12 e sindrome hipertensiva em 8 casos. Todavia, em 117 conceptos não foi possivel esclarecer a etiologia do óbito.

Conclusões: a morte fetal esteve presente notadamente nas primigestas (40,74\%) internadas no pré-termo (59,79\%), e ocorreram no periodo anteparto (86,31\%). Dentre as causas de morte fetal, a mais encontradiça foi o DPP (18,42\%) e em $61,57 \%$ dos casos ela não pôde ser determinada.
\end{abstract}

PALAVRAS-CHAVE: Óbito fetal. Descolamento prematuro da placenta. Malformações fetais. Hipertensão.

\section{Introdução}

Não obstante as divergências da literatura médica quanto à definição de óbito fetal, a maioria dos autores o reconhece como sendo aquele em que o concepto morre antes da completa extração ou expulsão do corpo materno, a partir de 20 semanas completas de gestação e/ou peso fetal superior a $500 \mathrm{~g}$.

Departamento de Obstetrícia da Universidade Federal de São Paulo - Escola Paulista de Medicina

Correspondência:

Nestor Nurdan

Praça Cesário Alvim, 98 - sala 10 - centro

35300-096 - Caratinga - MG

Fone/fax: (33) 3321-4949

e-mail: nestornurdan@uol.com.br
Alguns fatores de risco têm sido associados com a morte fetal. Certo número deles podem ser prevenidos e tratados. Contudo, em muitos casos de perda fetal nenhum fator pode ser detectado claramente. Muitos estudos de óbito fetal dão a falsa impressão de que os fatores atuam de maneira isolada e independente no desencadeamento da morte. Entretanto, na maioria das vezes, existe inter-relação, com atuação simultânea de diversos fatores ${ }^{1,2}$.

Destacaram-se em diversos estudos, os seguintes fatores mais fortemente associados à perda fetal: idade materna superior a 35 anos, nuliparidade, perdas fetais anteriores, doenças prévias como a hipertensão arterial e diabete melito, gemelaridade, anomalias cromossômicas fetais, tabagismo, infecções bacterianas e 
viróticas, ausência ou má qualidade da assistência pré-natal e deficiência no acompanhamento intraparto ${ }^{2,3}$.

Quando ocorre a morte fetal é importante a identificação da causa, não só para o esclarecimento dos pais, mas também para o planejamento de futura gestação. Partindo de diferentes classificações muitas causas são listadas na literatura. As mais freqüentemente citadas são malformações congênitas, baixo peso ao nascer, hemorragia anteparto e intraparto, anóxia/asfixia, acidentes do funículo, síndrome hipertensiva, infecção e diabete. Contudo, em 12 a 50\% dos casos a morte permanece inexplicável, mesmo após exaustiva pesquisa complementar ${ }^{4-6}$.

Considerando-se a análise do óbito fetal fundamental na promoção das ações voltadas para a saúde materno-infantil, objetivamos com este estudo analisar os fatores associados à ocorrência de morte fetal e identificar suas prováveis causas, em cidade do interior de Minas Gerais, longe de centros universitários.

\section{Pacientes e Métodos}

Trata-se de estudo epidemiológico descritivo em microrregião de Minas Gerais, que inclui 10 cidades próximas. Sendo Caratinga a cidade mais importante da região investigada, recebe para internação hospitalar todos os casos nos seus dois únicos hospitais (Casa de Saúde Divino Espírito Santo e Hospital Nossa Senhora Auxiliadora). A cidade situa-se em mesorregião do Vale do Rio Doce, distante aproximadamente $300 \mathrm{~km}$ de Belo Horizonte, com população estimada pelo IBGE (2000) em 77.732 habitantes. O contingente populacional de todas as cidades de abrangência da pesquisa é de 166.275 pessoas.

Inicialmente investigou-se o arquivo do único cartório de registro de nascimentos e óbitos (Cartório Fernando Patrício) de Caratinga, no período de $1^{\circ}$ de janeiro de 1995 a 30 de abril de 2000 . A seguir, todos os prontuários médicos das gestantes que parturiram nos dois únicos hospitais da cidade no mesmo período foram analisados.

Dentre as variáveis estudadas, quatro merecem destaque pela importância no presente estudo: número de gestações, idade gestacional no momento da internação hospitalar, ocorrência do decesso fetal em relação ao parto e causa do óbito fetal.

Quanto ao número de gestações, as grávidas foram divididas em primigestas ( $1^{\mathrm{a}}$ gestação), multigestas ( $2^{\mathrm{a}}$ a $5^{\mathrm{a}}$ gestação), grandes multigestas (mais de 5 gestações) e não apurada. Em relação à idade gestacional no momento da internação hos- pitalar, os casos foram separados em quatro grupos: 20 semanas completas a 37 semanas incompletas, 37 semanas completas a 42 semanas incompletas, maior ou igual a 42 semanas e não apurada. No que concerne ao parto, a morte fetal foi disposta em anteparto, intraparto e não apurada. Finalmente, classificou-se o determinante da perda fetal em causas não identificadas, maternas e feto-anexiais. É importante esclarecer que o diagnóstico da causa do decesso fetal foi realizado tão somente por critérios clínicos.

Não havendo grupos controle, optamos pela utilização de estatística descritiva com tabelas, figuras, porcentagens e médias aritméticas no estudo.

A pesquisa foi previamente analisada e aprovada pelos membros dos Conselhos de Ética dos hospitais envolvidos.

\section{Resultados}

No período de $1^{\circ}$ de janeiro de 1995 a 30 de abril de 2000, ocorreram 11.948 nascimentos em 11.825 partos, com 123 gemelares, na Casa de Saúde Divino Espírito Santo e Hospital Nossa Senhora Auxiliadora, Caratinga, MG. Foram encontradas 190 perdas fetais entre 189 gestantes (1 gemelar) na amostra analisada, no mesmo período.

No que diz respeito ao número de gestações das pacientes com morte fetal, houve predomínio do evento nas primigestas $(40,74 \%)$, seguido das grávidas com duas a cinco gestações $(40,21 \%)$ (Tabela 1).

Tabela 1 - Número de gestações das pacientes com morte fetal, registradas nos dois hospitais de Caratinga (MG), no período de janeiro de 1995 a abril de 2000.

\begin{tabular}{lrr}
\hline $\begin{array}{c}\text { Número de gestações } \\
\text { prévias }\end{array}$ & $\mathbf{n}$ & $\%$ \\
\hline 1 & 77 & 40,7 \\
2 a 5 & 76 & 40,2 \\
Mais de 5 & 20 & 10,5 \\
Não apurada & 16 & 8,4 \\
Total & 189 & 100 \\
\hline
\end{tabular}

A menor idade gestacional encontrada foi 21 semanas, a maior 43, sendo a média gestacional de 32,5 semanas. Evidencia-se, portanto, que o maior percentual de casos $(59,79 \%)$ era de gravidez de pré-termo (Tabela 2).

A ocorrência de decesso fetal em relação ao parto foi predominantemente no período anteparto, ocorrendo em 86,31\%. Houve 23 óbitos (12,1\%) no período intra-parto, e em 3 casos $(1,5 \%)$ não foi possivel apurar o exato momento da morte. 
Tabela 2 - Idade gestacional no momento da internação hospitalar, nos casos de perdas fetais, registradas nos dois hospitais de Caratinga (MG), no período janeiro de 1995 a abril de 2000

\begin{tabular}{lrr}
\hline Idade gestacional & $\mathbf{n}$ & $\mathbf{\%}$ \\
\hline $20-37$ semanas & 113 & 59,7 \\
$37-42$ semanas & 73 & 38,6 \\
$>42$ semanas & 02 & 1,0 \\
Não apurada & 01 & 0,5 \\
Total & 189 & 100 \\
\hline
\end{tabular}

$\mathrm{Na}$ avaliação das causas da morte fetal, observa-se predominância nítida das causas desconhecidas $(61,57 \%)$ e DPP $(18,42 \%$ dos casos). Dentre as causas maternas, evidenciaram-se a sindrome hipertensiva em 4,2\% e diabete melito, epilepsia e doença hemolitica perinatal em $0,5 \%$ dos casos, respectivamente. Dentre as causas fetoanexiais, o DPP foi a mais incidente $(1,4 \%)$, seguido pela anomalia fetal $(6,3 \%)$, infecção $(2,1 \%)$, insuficiência placentária $(2,1 \%)$, sofrimento fetal $(1,5 \%)$, acidente de funículo $(1 \%)$ e hidropisia $(0,5 \%)$ (Tabela 3).

Tabela 3 - Causas das perdas fetais, nos casos registrados nos dois hospitais de Caratinga (MG), no período de janeiro de 1995 a abril de 2000 .

\begin{tabular}{lrr}
\hline Causas das perdas fetais & $\mathbf{n}$ & $\%$ \\
\hline Desconhecida & 117 & 61,5 \\
DPP & 35 & 18,4 \\
Anomalia fetal & 12 & 6,3 \\
Síndrome hipertensiva & 8 & 4,2 \\
Infecção & 4 & 2,1 \\
Insuficiência placentária & 4 & 2,1 \\
Sofrimento fetal & 3 & 1,5 \\
Acidente de funículo & 2 & 1,0 \\
Acidente automobilístico & 1 & 0,5 \\
Diabete melito & 1 & 0,5 \\
Epilepsia & 1 & 0,5 \\
Hidropsia & & 0,5 \\
Doença hemolítica perinatal & 1 & 0,5 \\
Total & 190 & 100 \\
\hline
\end{tabular}

\section{Discussão}

A morte fetal, apesar da sua reconhecida importância no planejamento de ações preventivas em saúde pública, tem sido pouco estudada no Brasil e outros países. Os poucos trabalhos existentes na literatura estão relacionados a grandes cidades, notadamente a centros universitários. Esta pesquisa tem o mérito de investigar o óbito fetal em microrregião do interior mineiro que retrata a realidade da maioria das localidades brasileiras, sem universidades e/ou medicina de alta complexidade. Outrossim, este estudo é abrangente, desde que retrata a realidade da assistência obstétrica local, pois inclui todos os casos dos dois únicos hospitais disponíveis da região.

No que concerne ao número de gestações, verificamos predomínio de decesso fetal entre as primigestas $(40,74 \%)$ e percentagem significativa $(10,58 \%)$ entre as grávidas após a quinta gestação. Estes números são compatíveis com a literatura médica, que mostra risco obstétrico aumentado na primeira gravidez, talvez pela maior incidência de complicações obstétricas não diagnosticadas durante o pré-natal. Há melhora nas gestações subseqüentes, agravandose após a quinta gravidez. Nas grandes multigestas existe maior freqüência de intercorrências clínicas como diabete e hipertensão arterial; maior freqüência de intercorrências obstétricas como placenta prévia e DPP, e também menor adesão ao pré-natal porque estas mães têm diversos afazeres domésticos e outras crianças $^{3,7,8}$.

Quanto à idade gestacional no momento da parturição do natimorto, destacamos a ocorrência predominante entre 20 e 37 semanas, com média gestacional de 32,5 semanas. Portanto, o óbito fetal incidiu principalmente no pré-termo $(59,7 \%)$. O feto pré-termo tem maior risco para a morte, pela maior ocorrência de síndromes hemorrágicas, préeclâmpsia grave, insuficiência placentária, malformações e síndrome dos anticorpos antifosfolipí$\operatorname{deos}^{9,10}$.

Em relação ao trabalho de parto, evidenciamos a ocorrência de morte conceptual, principalmente no período anteparto $(86,2 \%)$. Estas taxas são superiores às retratadas por outros autores, mas é bom lembrarmos que nem sempre é fácil estabelecer o exato momento em que se deu a perda fetal ${ }^{4,11}$.

Entre as causas da morte, a análise da amostra evidenciou percentagem elevada de causas desconhecidas $(61,5 \%)$. Das causas explicáveis as mais freqüentes foram o DPP $(18,4 \%)$, anomalia fetal $(6,3 \%)$ e sindrome hipertensiva $(4,2 \%)$.

Estima-se que em 12 a $50 \%$ dos casos de perdas fetais a causa mortis não pode ser determinada claramente, mesmo após exaustiva propedêutica subsidiária. A maioria dos autores reconhece que a necropsia do feto e estudo histopatológico da placenta representam os exames mais importantes na elucidação da etiologia da morte. Lamentavelmente, na região estudada, a necropsia é de dificil execução, não só pela resistência dos pais em autorizá-la, como também pela baixa confiabilidade dos seus resultados, desde que 
ela é realizada por médicos legistas, mais afeitos ao estudo de mortes violentas ${ }^{12,13}$.

A literatura obstétrica tem chamado atenção ao ominoso prognóstico fetal no DPP. Assinala que nas últimas décadas, apesar do avanço da Obstetrícia, esta doença continua ser grande causadora de óbito perinatal. Enfatiza que torna-se imperioso tomar medidas profiláticas, principalmente durante o pré-natal, antes da ocorrência do DPP. Então, é importante separar e atuar nas gestantes de risco: multiparas, tabagistas, DPP anterior, idosas, baixo nivel socioeconômico e principalmente as hipertensas crônicas, que respondem por $50 \%$ dos casos.

No que tange à anomalia fetal, as aberrações cromossômicas são as mais encontradiças, representadas principalmente pela sindrome de Down. Cerca de 5\% das gestações a termo apresentam fetos com alterações morfológicas e/ou funcionais. As principais indicações para rastreio das anomalias fetais são a idade materna avançada, antecedentes de doenças hereditárias, crescimento fetal restrito, gravidez múltipla, consangüinidade, oligoâmnio e polidrâmnio. Ressalte-se, contudo, que o aconselhamento pré-concepcional nas gestantes de risco para fetos anômalos é o melhor caminho para a profilaxia de perdas fetais decorrentes $^{9,11}$.

Em referência à síndrome hipertensiva da gravidez (representada pela doença hipertensiva específica da gravidez, hipertensão arterial crônica ou hipertensão arterial crônica com préeclâmpsia associada), esta constitui o fator de risco mais importante para morte fetal em todo o mundo. Se não existem medidas profiláticas para a prevenção primária da pré-eclâmpsia, o pré-natal bem conduzido deve prevenir as formas graves de eclâmpsia. Reconhecendo-se de risco as primigestas, hipertensas crônicas, pacientes com antecedentes de pré-eclâmpsia grave, diabéticas com vasculopatias, gravidez múltipla e novo parceiro na gestação atual, esses casos poderiam ser conduzidos para melhor prognóstico maternofetal $^{14,15}$.

Em nossa opinião, é importante que a análise do óbito materno e fetal, em regiões do interior do Brasil, seja realizada continuamente. Porque, assim, subsídios importantes são oferecidos às autoridades ligadas à Saúde Pública e à própria comunidade local, na identificação dos problemas e no direcionamento das ações prioritárias em saúde reprodutiva.

\section{ABSTRACT}

Purpose: to study the causes and associated factors of fetal death.

Methods: epidemiological descriptive study, composed of 190 cases of fetal loss amongst 11,825 pregnant women that gave birth at the two only hospitals (Casa de Saude Divino Espirito Santo and Hospital Nossa Senhora Auxiliadora) of Caratinga City, in the State of Minas Gerais, Brazil, in the period from January 1, 1995 to April 30, 2000. The variables were the number of pregnancies, the timing of the pregnant women at the time of hospitalization, the occurrence of fetal death in relation to delivery and the cause of fetal death. Since there were no comparative groups, tables, percentages and arithmetical means were applied, following the guidelines of the Course of Statistics, of the "Universidade Federal de São Paulo".

Results: among the 189 pregnant women with fetal death, 77 were primigravidal and 76 had already been pregnant 2 to 5 times. The gestational age in 113 women was from 20 to 37 weeks. In relation to parturition, the fetal loss occurred during the antepartum period in 164 of 190 dead fetuses. The most frequently noticed death causes were: abruptio placentae in 35 cases, fetal anomaly in 12 cases, and hypertension syndrome in 8 cases. Nervertheless, there was no explanation for the etiology of 117 cases of fetal death. Conclusion: stillbirth has frequently been observed among the primigravidae (40.74\%), in preterm period $(59.79 \%)$, and in the antepartum period (86.31\%). Among fetal death causes, the most frequent was a abruptio placentae (18.42\%), and in $61.57 \%$ of the cases the fetal death could not be explained.

KEYWORDS: Fetal death. Abruptio placentae. Fetal anomaly. Hypertension.

\section{Referências}

1. Walles B, Tyden T, Herbst A, Ljungblad U, Rydhstrom H. Maternal health care program and markers for late fetal death. Acta Obstet Gynecol Scand 1994; 73:773-8.

2. Aquino, MMA, Cecatti JG, Mariani Neto C. Risk factors associated to fetal death. São Paulo Med J 1998; 116:1852-7.

3. Conde-Agudelo A, Belizán IM, Diaz-Rossello JL. Epidemiology of fetal death in Latin America. Acta Obstet Gynecol Scand 2000; 79:371-8.

4. Ahlenius I, Floberg J, Thomassen P. Sixty-six cases of intrauterine fetal death. A prospective study with an extensive test protocol. Acta Obstet Gynecol Scand 1995; 74:109-17. 
5. Incerpi MH, Miller DA, Samadi R, Settlage RH, Goodwin TM. Stillbirth evaluation: what tests are needed? Am J Obstet Gynecol 1998; 178:1121-5.

6. Huang DY, Usher RH, Krammer MS, Yang H, Morin L, Fretts RC. Determinants of unexplained antepartum fetal deaths. Obstet Gynecol 2000; 95:215-21.

7. Hsieh HL, Lee KS, Khoshnood B, Herschel M. Fetal death rate in the United States, 1979-1990: trend and racial disparity. Obstet Gynecol 1997; 89:33-9.

8. Costa CFF, Costa HLFF, A Júnior H, Machado KMM, Cunha MEG. Óbito fetal: estudo de 105 casos. Rev IMIP 1999; 13:22-6.

9. Finan A, Clarke TA, Matthews TG, et al. Strategies for reduction of neonatal mortality. Ir $\mathrm{J}$ Med Sci 1999; 168:265-7.
10.Sheiner E, Hallak M, Shoham-Vardi I, Goldstein D, Mazor M, Katz M. Determining risk factors for intrapartum fetal death. J Reprod Med 2000; 45:419-24.

11.Fretts RC, Usher RH.. Causes of fetal death in women of advanced maternal age. Obstet Gynecol 1997; 89:40-5.

12.Saller DN Jr, Lesser KB, Harrel U, Rogers BB, Oyer $\mathrm{CE}$. The clinical utility of the perinatal autopsy. JAMA 1995, 273:663-5.

13.Faye-Petersen OM, Guinn DA, Wenstrom KD. Value of perinatal autopsy. Obstet Gynecol 1999; 94:915-20.

14. Costa SM, Ramos JG, Berger CB, Goldim JR. Fatores de risco para natimortalidade em gestantes hipertensas. J Bras Ginecol 1990; 100:287-90.

15. Cecatti JG, Aquino MMA. Causas e fatores associados ao óbito fetal. Rev Ci Med 1998; 7:43-8.

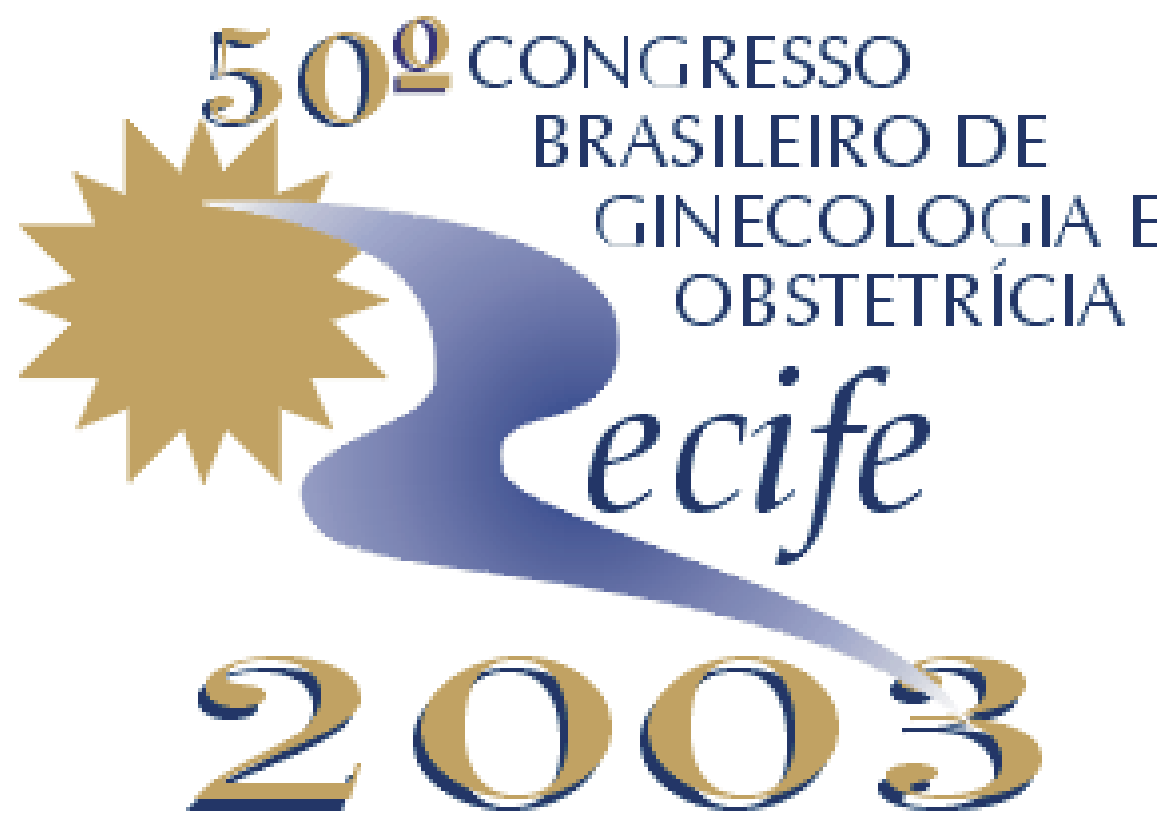

18 a 22 de novembro INFORMAÇÖES: Telefone: (81) 3222-5112 E-mail: sogope1@terra.com.br 